\title{
Production and tests of Hybrid Photon Detectors for the LHCb RICH detectors
}

\author{
Stephan Eisenhardt ${ }^{1}$ \\ School of Physics \& Astronomy, University of Edinburgh, Mayfield Road, Edinburgh EH9 3JZ, UK
}

\section{A R T I C L E I N F O}

Available online 20 July 2008

\section{Keywords:}

Hybrid Photon Detector

Silicon pixel sensor

Ring Imaging Cherenkov (RICH) counter

\begin{abstract}
A B S T R A C T
The LHCb Ring Imaging Cherenkov (RICH) detectors employ 484 pixel Hybrid Photon Detectors (HPDs) as a photo-sensitive device. A total of 559 HPDs were produced over the course of two years and subjected to an exhaustive quality-assurance programme to verify that the operation meets specifications. Results of tests of the full production sample are presented and compared to expectations.
\end{abstract}

(c) 2008 Elsevier B.V. All rights reserved.

\section{Introduction}

The LHCb experiment $[1,2]$ requires an excellent separation of $\pi$ and $\mathrm{K}$ mesons over the momentum range of $1-100 \mathrm{GeV} / c$. This is achieved by a combination of two Ring Imaging Cherenkov (RICH) detectors [2,3]. The chosen photon detector is the pixel Hybrid Photon Detector (HPD) [4,5], covering an area of $\sim 3.3 \mathrm{~m}^{2}$ with a granularity of $2.5 \times 2.5 \mathrm{~mm}^{2}$. The HPDs provide sensitivity to single photons in the wavelength range of $200-600 \mathrm{~nm}$, a typical quantum efficiency (QE) of $23 \%$ at $270 \mathrm{~nm}$, an active area fraction of $\sim 65 \%$ and tolerance to radiation of up to $\sim 3 \mathrm{krad}$ per year. A total of $0.5 \mathrm{M}$ channels are read out at $40 \mathrm{MHz}$.

The pixel HPD is a vacuum photon detector tube equipped with an anode assembly comprising a pixelised silicon sensor and readout chip [6,7]. An S20 photo-cathode of diameter $72 \mathrm{~mm}$ is deposited on the inside surface of a $7 \mathrm{~mm}$ thick quartz entry owindow. Photo-electrons are accelerated onto the Si-sensor by a Cross-focusing electrostatic field which has a demagnification factor of five, producing about 5000 electron-hole pairs at an operating Voltage of $20 \mathrm{kV}$. The Si-sensor is segmented into an array of $256 \times$ ○32 pixels of size $62.5 \times 500 \mu \mathrm{m}$. The sensor is bump-bonded to the readout chip which provides a preamplifier, a shaper and a discriminator circuit for each pixel, giving a binary readout.

The results on tests of nine pre-series HPDs have been described in Ref. [8]. The results from production and testing of the full sample (559 HPDs) are summarised below.

\section{Photon-detector test procedures}

To characterise and qualify the HPDs after production at the manufacturer Photonis-DEP, ${ }^{2}$ LHCb set up two Photon Detector Testing Facilities (PDTF), described in Ref. [8], at the Universities of

\footnotetext{
E-mail address: S.Eisenhardt@ed.ac.uk

${ }^{1}$ On behalf of the LHCb RICH Collaboration.

2 Photonis Netherlands B.V., 9301 ZR Roden, Netherlands.
}

Edinburgh and Glasgow. The plan was to test one HPD per day per site to cope with the production rate of 30 HPDs per month. On average the standard preparation and automated test programme took $6 \mathrm{~h}$ per HPD. The testing procedure quantified every function and property of the HPD. These tests can be grouped into five areas: parameters characterising the photocathode, the electron optics, the state of the tube body and exterior, the characteristics of the silicon sensor and the functionality of the readout chip. In order to provide reliable and comparable test results in minimum time, the test programme was automated as much as possible. The parameter settings, data taking and logging, the data analysis and report generation were executed by the readout computer. Human control was required for the choice of a few key parameters, the monitoring of the online displays, if necessary for interventions, and finally for the interpretation of offline reports.

Altogether 559 HPDs were produced and tested. The whole test programme stretched over 21 months. Production and test rates of up to $40 \mathrm{HPDs}$ per month were achieved, while maintaining the high standards of tube qualification.

When not in use, the tubes were stored in a helium-free atmosphere under nitrogen flow to minimise deterioration of the tube vacuum by diffusion of helium through the quartz window and sintered aluminium body.

\section{HPD test results}

By the end of the testing period, a total of 547 out of 559 HPDs passed the qualification procedure, i.e. 98\% of the sample, and only 12 HPDs had to be rejected. In the following, a selection of detailed test results is presented.

Firstly the HPDs were subjected to tight mechanical tests. Using a precision jig with two clamps closing around a mounted tube, the uprightness and sphericity of the tube body were tested. The closed jig allowed for a $0.1 \mathrm{~mm}$ margin around the tube and contact with the jig was regarded as failure. This was to ensure that the HPDs will fit in a close-packing arrangement when 
integrated into the RICH system. Two HPDs which initially failed this test were repaired and subsequently qualified.

The functionality of the readout chip was then characterised. The global threshold of the readout chip of each HPD was set as low as possible but high enough to suppress the electronic noise of each of the 8192 readout channels of the sensor array. Fig. 1 shows the distribution of the global threshold across the full HPD production sample. The average threshold, $\langle T\rangle$, of $1065 \mathrm{e}^{-}$, is significantly below the specification of $<2000 \mathrm{e}^{-}$.

The two main contributions to the noise of each readout channel are the capacitive noise of the sensor pixel and the electronic noise of the preamplifier. By varying the global readout threshold, the noise per pixel could be measured. The distribution of the noise per pixel, averaged over each HPD, is displayed in Fig. 2. The average over the full HPD production sample, $\langle N\rangle$, is $145 \mathrm{e}^{-}$, far below the specification of $<250 \mathrm{e}^{-}$.

With the average signal size $\langle C\rangle=5000 \mathrm{e}^{-}$, we define an average signal-over-noise ratio as $\langle S / N\rangle=(\langle C\rangle-\langle T\rangle) /\langle N\rangle=27$. This excellent $S / N$ ratio is achieved without the use of an option provided by the readout chip to optimise the sensitivity to signal by manually adjusting the threshold of each individual readout channel using a 3-bit register. The excellent $S / N$ is essential for the detection of photo-electrons which are scattered back off the sensor surface and thus deposit only a fraction of the kinetic energy gained in the electrostatic field.

With $\mathcal{O}(10 \mathrm{k})$ per HPD bump-bonds subjected to a bake-out cycle at $300^{\circ} \mathrm{C}$ during the vacuum tube production, the channel yield of an assembly was of particular importance. The requirement was that more than $95 \%$ of the 8192 pixels are fully operational. It was found that most HPDs had less than 20 dead pixels and that the number of noisy pixels per HPD was negligible.

During the test procedure, an HPD was first allowed to settle for about $3 \mathrm{~h}$ after the initial high voltage ramp-up before the dark count rate was measured in a high statistics run (5M triggers). All accepted HPDs had a photocathode dark count rate of less than $20 \mathrm{kHz} / \mathrm{cm}^{2}$ and 497 HPDs yielded a dark count rate of less than $5 \mathrm{kHz} / \mathrm{cm}^{2}$. The latter rate corresponds to a $1 \%$ probability of having one hit per HPD per event during LHC operation. The distribution of dark count rate per HPD is shown in Fig. 3. The higher rates in the tail of the distribution are caused by two types of dark counts: an increased red sensitivity of the photocathode, which exhibits a uniform distribution of hits across the photocathode image, and an increased probability of ion feedback,

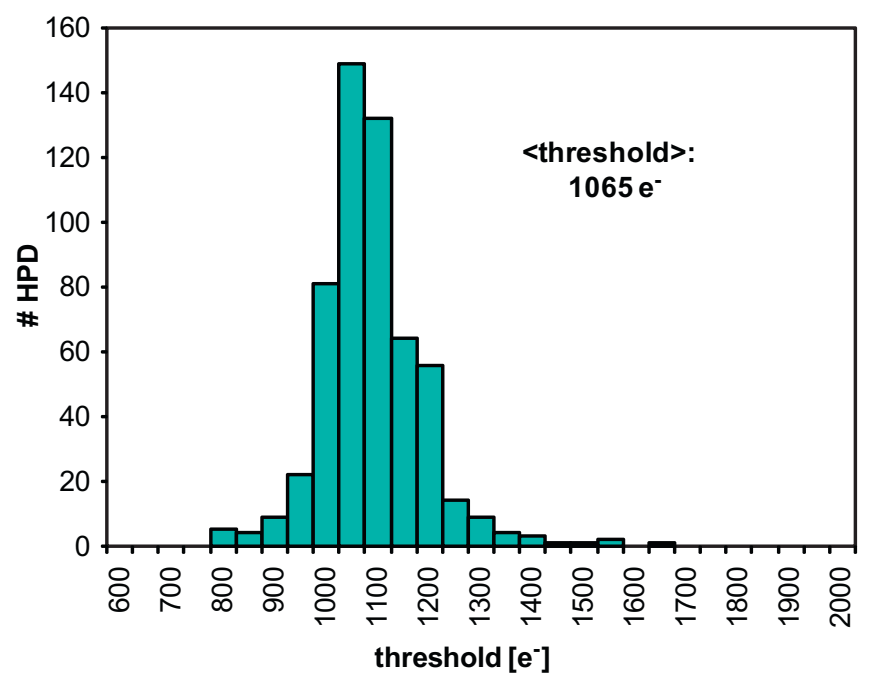

Fig. 1. The global thresholds of the HPD readout chips.

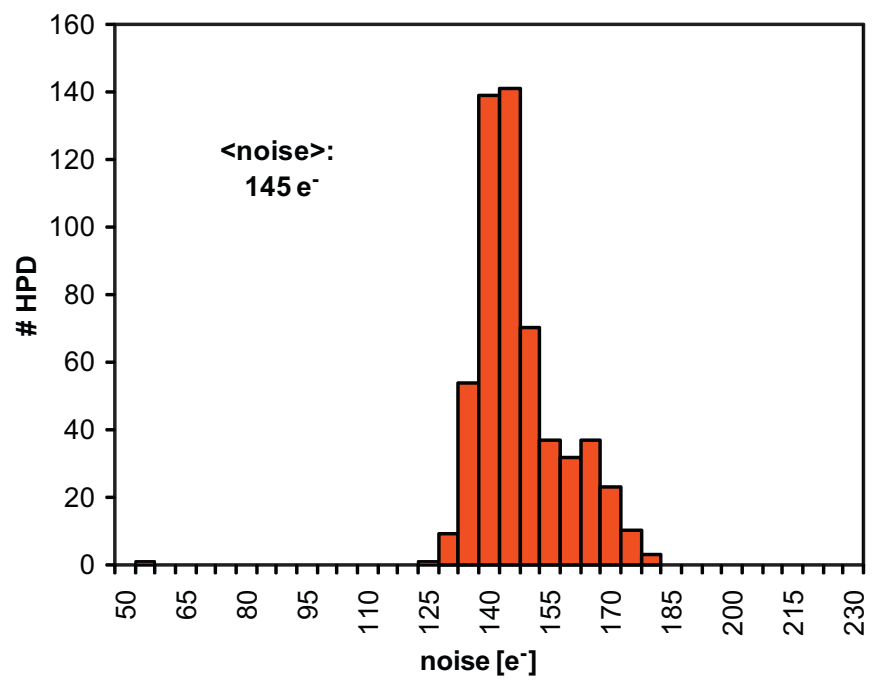

Fig. 2. Noise of readout pixels, averaged over each HPD.

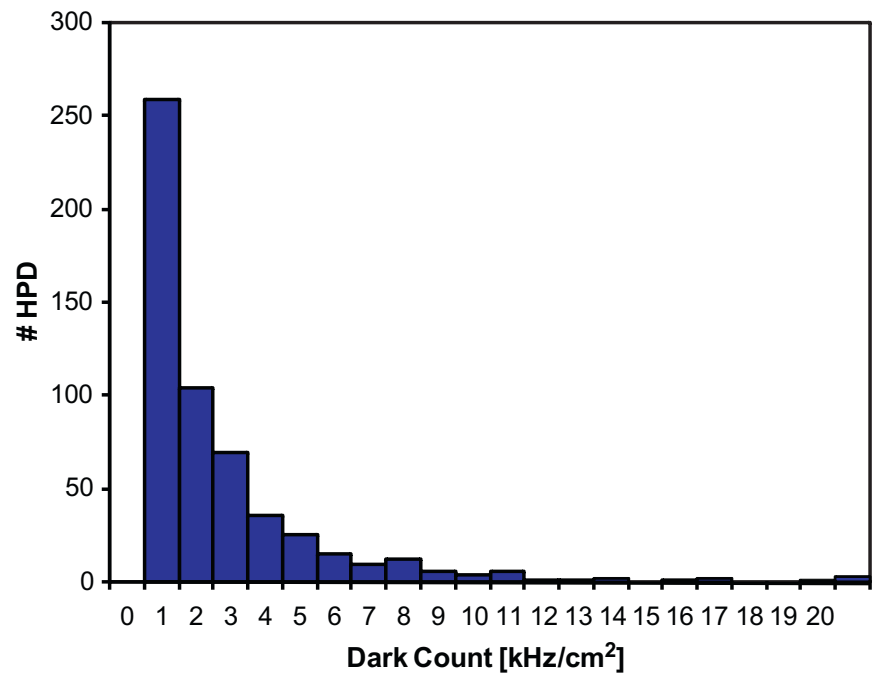

Fig. 3. The dark count rate per HPD.

which leads to large clusters of hits concentrated at the centre of the image.

Ion feedback occurs when a photo-electron ionises a residual gas atom in the vacuum tube. The ion drifts back to the photocathode and releases secondary photo-electrons, typically about 20. At the Si-sensor the secondaries form clusters of hits. A clustering algorithm combines the hits event-by-event and identifies clusters of five or more hits as ion feedback. The delay of these clusters relative to the primary photo-electron, typically 200-300 ns, is governed by the drift time of the ion. Fig. 4 shows a typical measurement of photo-electron and ion feedback hits as a function of the delay between light pulse and the trigger signal to the HPD. About $250 \mathrm{~ns}$ after the direct photo-electron signal, the ion feedback from large clusters shows as a small peak, made visible by being scaled up by a factor of 100 . Fig. 5 shows the measured ion feedback fraction for the full HPD production sample. The average ion feedback fraction is $0.04 \%$ compared to the signal of direct photo-electrons. This leads to the conclusion that the tube vacuum at fabrication is very good.

The QE of the photocathode was measured by the manufacturer for each HPD in the wavelength range 200-900 nm. Fig. 6 presents the $\mathrm{QE}$ measurements as a function of delivery batch. 


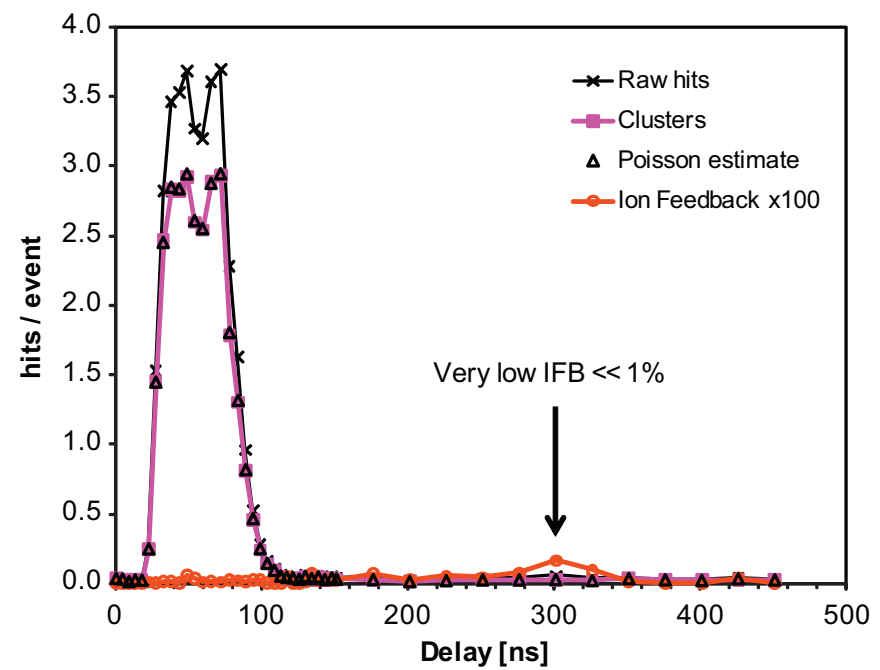

Fig. 4. Measured rate of photo-electron and large cluster hits (the ion feedback fraction is multiplied $\times 100$ ) for various delays between light pulse and the trigger signal to the HPD.

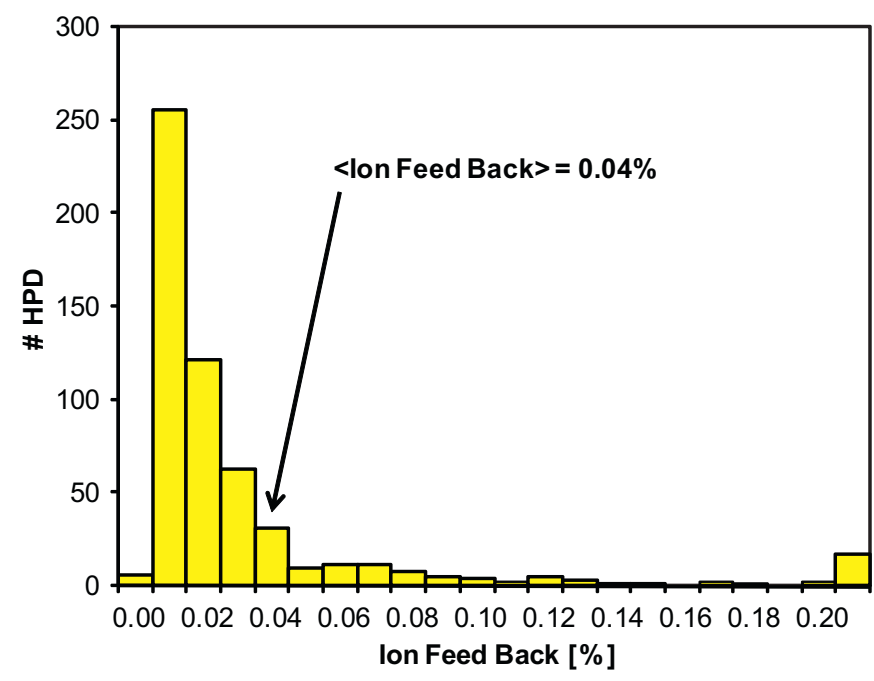

Fig. 5. Measured ion feedback fraction.

This shows that the QE gradually improved during the production in three ways: the peak sensitivity was increased, the gap between the UV and the visible was reduced and the red sensitivity at $800 \mathrm{~nm}$ was also reduced. Fig. 7 illustrates the improvement of the peak sensitivity at $270 \mathrm{~nm}$ over the production period. This improvement was achieved by tuning the photocathode production process. The total average of the peak $\mathrm{QE}$ at $270 \mathrm{~nm}$ is $30.8 \%$. The average integrated QE is $27 \%$ larger than expected from the pre-series HPDs.

Due to the importance of the $\mathrm{QE}$ for the $\mathrm{LHCb} \mathrm{RICH}$ performance, an independent QE measurement was performed at the PDTFs. Using a quartz-tungsten lamp with stabilised current and $10 \mathrm{~nm}$ bandpass filters, the QE was measured at seven wavelengths by comparing the photo-current to that of a calibrated photodiode. The measurement error was $2 \%$. A subsample of 76 HPDs, 15\%, was measured. Fig. 8 compares the Photonis-DEP and PDTF measurements of four representative HPDs. The two measurements typically agree within $3 \%$. This gives full confidence of the excellent QE performance of all production HPDs.

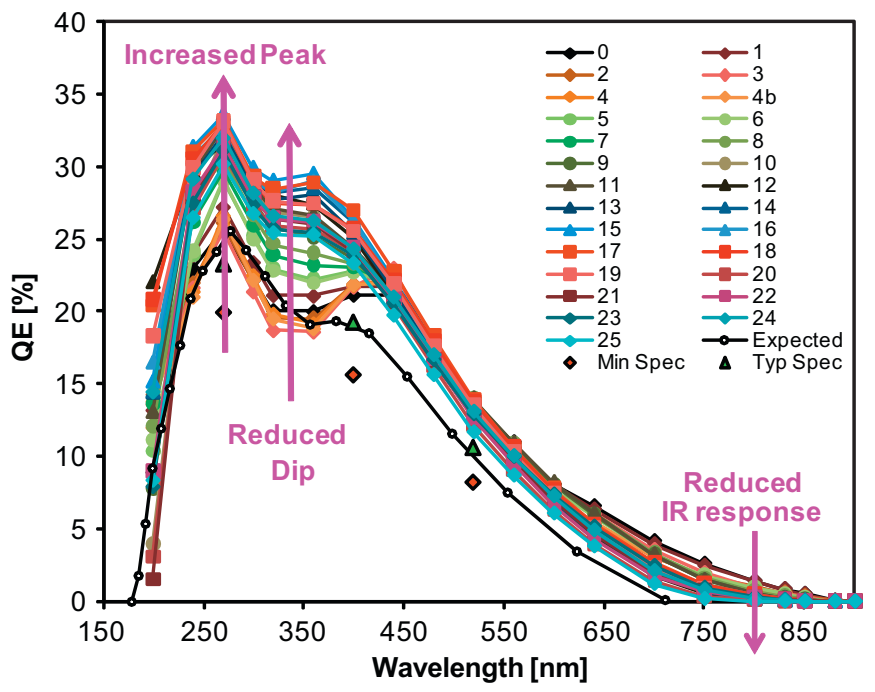

Fig. 6. QE of HPDs measured by Photonis-DEP as a function of wavelength, one curve per delivery batch. The arrows indicate production trends.

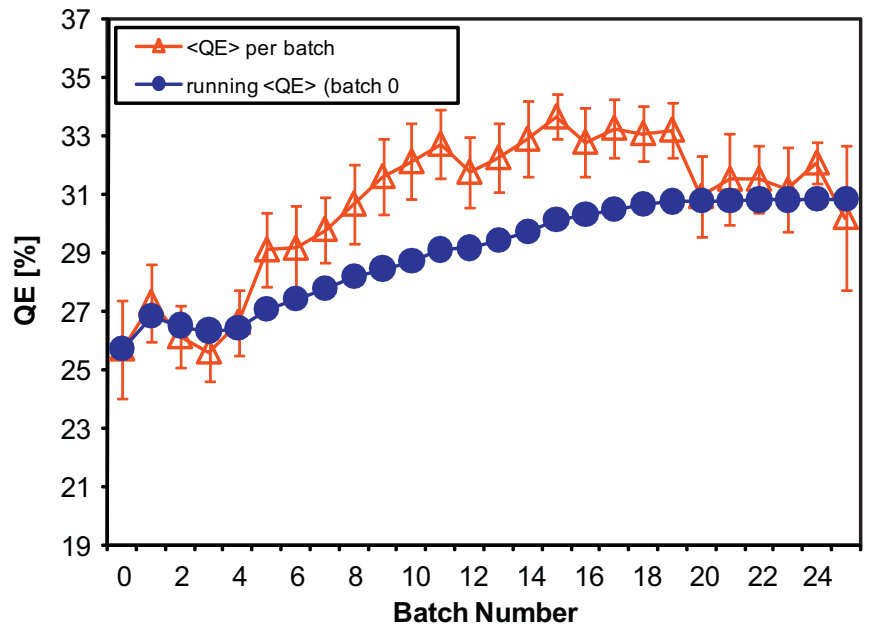

Fig. 7. QE of HPDs measured at $270 \mathrm{~nm}$ per delivery batch and its running average Errors indicate RMS spread.

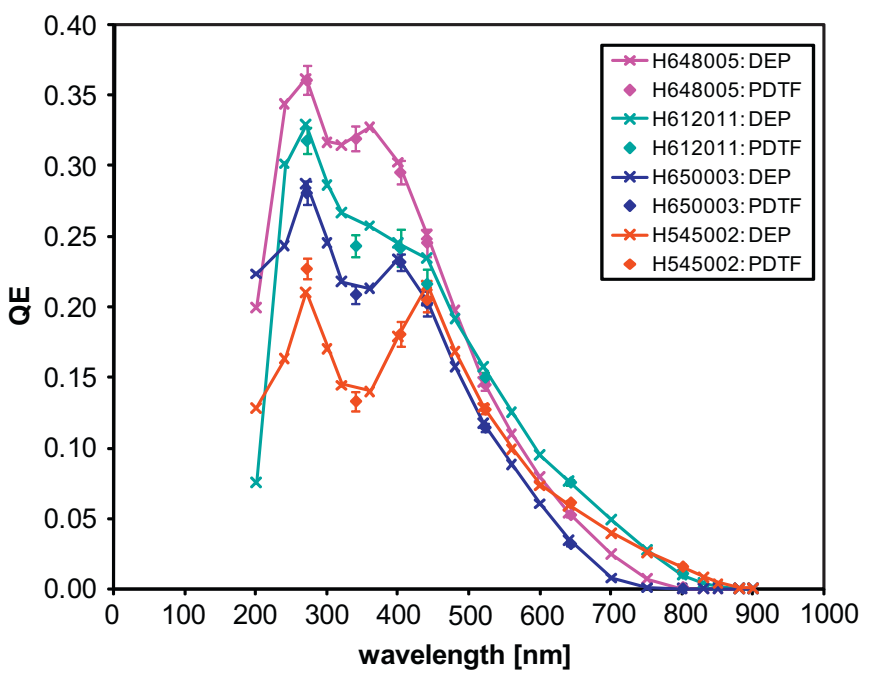

Fig. 8. Comparison of $\mathrm{QE}$ measurements for four typical HPDs. The lines are Photonis-DEP data, the dots with errors are PDTF data. 


\section{Conclusions}

The production and rigorous testing of 559 HPDs is now complete. Of these, $98 \%$ meet the requirements of the LHCb RICH detectors and have been accepted. The read-out sensors exceed specifications and exhibit an excellent $S / N$. The HPDs show a very good vacuum quality and very low dark count. The average $\mathrm{QE}$ surpassed expectations by $27 \%$ and the Photonis-DEP QE measurements were confirmed independently by LHCb. The commissioning of the LHCb RICH system is underway, with 288 HPDs installed in the RICH 2 detector.

\section{References}

[1] LHCb Technical Proposal, LHCC 98/04, LHCC/P4, February 1998.

[2] Technical Design Report, CERN/LHCC 2003-030, LHCb TDR 9, 9 September 2003.

[3] S. Amato, et al., CERN/LHCC/2000-0037, LHCb TDR 3, September 2000.

[4] M. Alemi, et al., Nucl. Instr. and Meth. A 449 (2000) 48.

[5] M. Moritz, et al., in: Proceedings of the 13th IEEE-NPSS Real Time Conference (RT 2003), Montreal, Canada, 18-23 May 2003, IEEE Trans. Nucl. Sci. NS-51 (2004) 1060.

[6] K. Wyllie, et al., in: Proceedings of the Fifth Workshop on LHC Electronics, Snowmass, CO, USA, 20-24 September 1999, CERN/LHCC/99-33.

[7] K. Wyllie, et al., Nucl. Instr. and Meth. A 546 (2005) 86.

[8] S. Eisenhardt, Nucl. Instr. and Meth. A 565 (2006) 234. 